\title{
Perylene-embedded electrospun PS fibers for white light generation
}

\author{
Tugrul Guner ${ }^{\mathrm{a}, 1}$, Erkan Aksoy ${ }^{\mathrm{b}, 1}$, Mustafa M. Demir ${ }^{\mathrm{a}, * *}$, Canan Varlikli ${ }^{\mathrm{c}, *}$ \\ a Department of Materials Science and Engineering, Izmir Institute of Technology, Izmir, Turkey \\ ${ }^{\mathrm{b}}$ Institute of Solar Energy, Ege University, Izmir, Turkey \\ c Department of Photonics, Izmir Institute of Technology, Izmir, Turkey
}

\section{A R T I C L E I N F O}

\section{Keywords:}

Perylene diimide

Solid state emission

Polystyrene fiber

Frequency down-conversion

Aggregation

\begin{abstract}
A B S T R A C T
Perylene dyes have been employed in the fabrication of white light due to their superior photophysical properties and relatively easy synthetic methods. However, their molecular aggregation in solid state is one of the main handicaps since it causes deviation in their optical properties and quenches photoluminescence quantum yields $\left(\Phi_{\mathrm{f}}\right)$. Investigation of the photophysical properties of a green (PTE), a yellow (PDI) and a new red (DiPhAPDI) emitting perylene derivative in solution, drop-casted films, polystyrene (PS) fibers and PS fibers embedded in poly (dimethyl siloxane) (PDMS) showed that PS:dye fibers prevent aggregation to some extend and allows high $\Phi_{\mathrm{f}}$ of dyes. The $\Phi_{\mathrm{f}}$ values of PTE, PDI and DiPhAPDI were all higher than $93.0 \%$ in solution and 84.8\%, 94.3\% and 73.6\%, respectively in PS:dye fibers. Embedding the fibers in PDMS improved the photostabilities of the dyes two folds compared to their solution phases. The prepared dye containing fibers were combined together into a single PDMS film and utilized as a frequency conversion layer on a blue LED. Fabricated samples were found to show high color rendering index ( $\geq 90$ ), adjustable CCT (7500 K-5000 K), and power efficiency values exceeding $2001 \mathrm{~m} / \mathrm{W}$ depending on the used fiber amount in mass.
\end{abstract}

\section{Introduction}

It is recognized that $20 \%$ of worldwide electricity production is consumed in lighting. Compared to an equivalent incandescent lamp, light emitting diodes (LEDs) may provide $80 \%$ energy saving [1,2]. In white light LED generation, two main strategies are followed; multichip configuration and phosphor conversion [3]. In the former, three main colors; red, green and blue are obtained from LED chips individually. Even though the resulting white light shows high Color Rendering Index (CRI) and low Correlated Color Temperature (CCT), this process involves a high cost and complex fabrication process [4,5]. The main reason of this complexity is the different driving voltage/current requirements of these individual chips. On the other hand, the latter case requires only a single blue or UV LED chip, and frequency conversion layer on top of it [6-8]. Although in both cases efficiency degrades as the LED heats up during operation and this can cause a color shift, frequency down-conversion is the most widely used approach because of the application simplicity it provides [2].

Frequency conversion layer mainly consists of inorganic phosphors or polymer/phosphor composites. A mainstream design of such a system contains a yellow phosphor, namely Cerium doped Yttrium Aluminum Oxide (YAG: $\mathrm{Ce}^{3+}$ ), and a blue LED chip which unfortunately suffers from high CCT and low CRI due to its deficiency of red emission region. In this sense, research on introduction of new rare-earth element containing phosphors $[9,10]$, rare-earth element free phosphors $[11,12]$, perovskites [13,14] and organic phosphors [15-20] is continued. Among those, organic phosphors are the promising materials due to their abundancy, absorption and photoluminescence (PL) wavelength tunability, and high PL efficiency.

Perylene diimides (PDI) and their derivatives are $\pi$-conjugated materials those can be used in photovoltaic devices, sensors, LEDs, and field effect transistors due to their strong visible range absorption, high PL quantum yield, high photostability and thermal durability [15-17,21-30]. However, the PL color of regular PDIs is limited with yellow region and their PL is quenched in their films and solid states due to intermolecular $\pi-\pi$ interactions. To overcome these problems, several methods including copolymerization approach [18], 3D functionalization [22,26,27,31,32], molecular isolation [33], or self-assembly monolayer formation [34] have already been attempted. All those attempts either limited the new structure with regular PDI

\footnotetext{
* Corresponding author.

** Corresponding author.

E-mail addresses: mdemir@iyte.edu.tr (M.M. Demir), cananvarlikli@iyte.edu.tr (C. Varlikli).

${ }^{1}$ Author contributions: First and second authors contribute equally to the manuscript. The manuscript was written through contributions of all authors. All authors have given approval to the final version of the manuscript.
} 


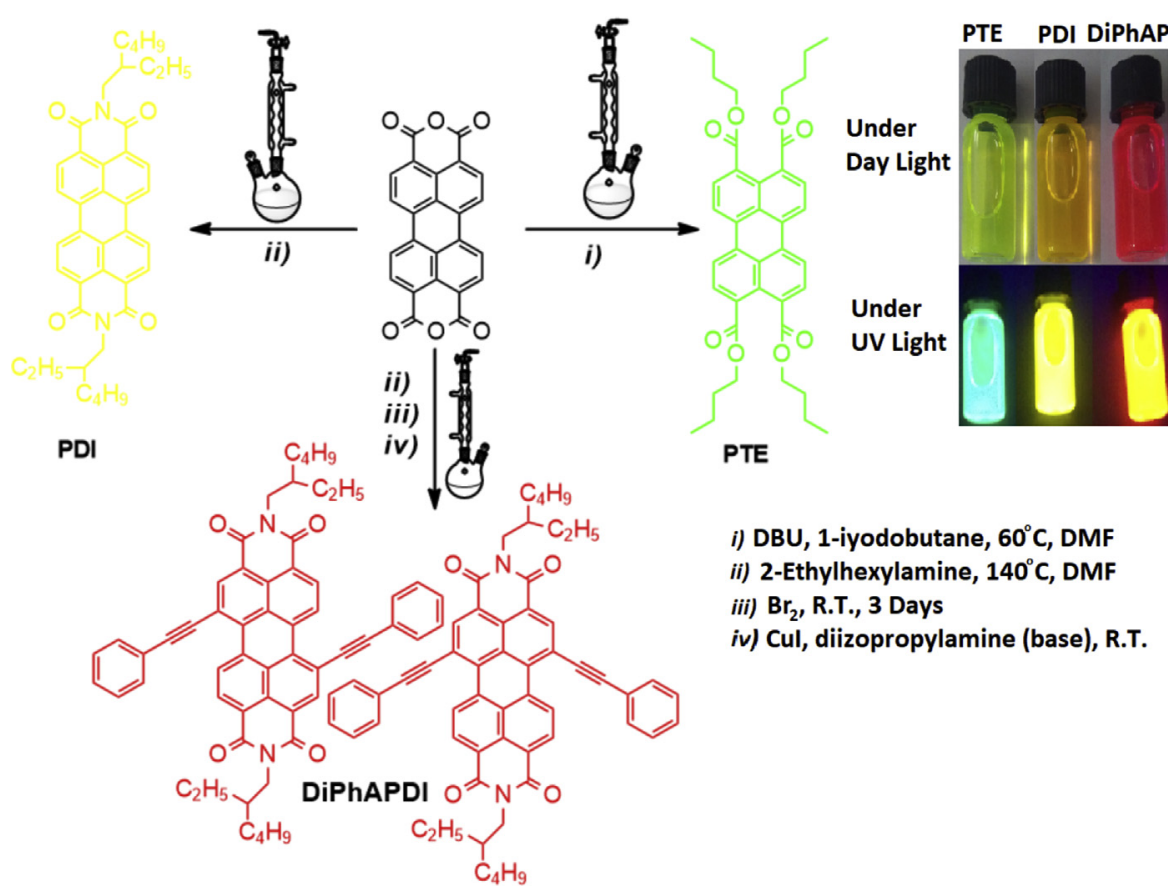

Scheme 1. Summary of synthetic path followed for PTE, PDI and DiPhAPDI and their visualization under daylight and UV illumination.

properties or caused casualties from the thermal and electrochemical stabilities and superior photophysical properties. Increasing the PL bandwidth of PDIs and the transfer of these molecules to a polymer system, while preventing them from aggregation at the same time, is a challenge in material science. Functionalization of PDIs at their bay positions through sp hybridized carbon atoms may prevent bending and increase their PL wavelength. Electrospinning appears to be an important method to overcome and control the formation of molecular aggregates, accordingly the optical feature of the composite system changes $[35,36]$. It is a straightforward technique to produce polymer fibers using a high potential difference between a polymer droplet and a collector to form jet. In this sense, both polymer and its solvent system must be determined carefully to allow the formation of fiber mat having mechanical integrity. To date, various polymer/solvent systems were reported to obtain fibers with $\mu \mathrm{m}$ or $\mathrm{nm}$ in diameter successfully [35-37]. Due to their high surface area fibers, can be employed in LED based white light applications since they are able to scatter light intensively by increasing the optical path length of the LED.

In this study, green, yellow and red emitting perylene derivatives were synthesized, and they were associated with polystyrene (PS) fibers. It was found that organic dye-embedded PS fibers are able to maintain the optical properties of these dyes and embedding them into fibers prevents the molecules from $\pi-\pi$ stacking to some extent. Then, the prepared perylene containing fibers were combined together in a single poly (dimethyl siloxane) (PDMS) film as frequency conversion layer and applied on a blue LED to obtain tri-chromatic white light source. As a result, by utilizing not only the extraordinary photo- and thermal properties of perylene derivatives but also thermal stability and optical advantages of PS [38] and PDMS [39], high CRI, low CCT color, and stable conversion layers were achieved over a blue LED chip. This method of organic dye embedded fibers presents an alternative to phosphor based color conversion layers.

\section{Experimental}

\subsection{Materials}

Potassium carbonate, 1-iodobutane and 1,8-diazabicyclo (5.4.0) undec-7-ene (DBU) were from Acros Organics, VWR Chemicals, ABCR
Chemicals and TCI Chemicals, respectively. Ethanol absolute, tetrakis (triphenylphosphine)palladium (0), phenylacetylene, hydrochloric acid, bromine, 1-butanol, methanol, acetonitrile (ACN), hexane, chloroform, dichlorometane (DCM), $N, N$-dimethyl-formamide (DMF), acetic acid, 1-methyl-2-pyrrolidinone, silica gel 0.040-0.063 mm were obtained from Sigma-Aldrich, diisopropylamine, silica gel 60 F254 (thin-layer chromatography) and 2-ethyl-1-hexylamine were obtained from Merck and perylene-3,4,9,10-tetracarboxylic dianhydride (PTCDA), copper(I) iodide were purchased from Fluka. Polydimethylsiloxane (PDMS, Sylgard-184, Dow Corning), toluene (Tol., $\geq 99.9 \%$, Sigma-Aldrich), tetrahydrofuran (THF, VWR, $\geq 99.7 \%$ ), and polystyrene (PS, Sigma-Aldrich) were purchased and used as received without any further purification.

\subsection{Methods}

${ }^{1} \mathrm{H}$ NMR spectra were recorded on a Bruker $400 \mathrm{MHz}$ spectrometer and Infrared (FTIR) spectra were obtained by using a PerkinElmer Spectrum BX-FTIR spectrophotometer. UV-Vis absorption measurements were performed by using Analytik Jena S 600 UV-Vis spectrophotometer. Photoluminescence (PL), absolute photoluminescence quantum yield $\left(\Phi_{\mathrm{PL}}\right)$ and life time $(\tau \mathrm{f})$ (EPL-470 excitation) spectra were recorded by using Edinburgh Instruments FLS920P spectrophotometer. Molecular models of the molecules were produced using Chem 3D pro v10 using its MM2 energy minimization routine. Thermal stability analyses were performed by Perkin Elmer STA 6000 analyzer. Fiber morphology was examined by Scanning Electron Microscopy (SEM; Quanta 250, FEI, Hillsboro, OR, USA). The white light properties were determined by using an integrating sphere (ISP-50-80-R, Ocean Optics Inc.) connected to a USB2000 + spectrometer (Ocean Optics Inc., Dunedin,FL, USA) via a premium fiber cable.

\subsection{Synthesis of organic dyes}

Synthetic procedure followed for the organic dyes with different colors; PTE [40] as green, PDI [41] as yellow, and DiPhA-PDI as red are summarized in Scheme 1 including their visualization under daylight and UV illumination. During the synthesis of PTE and PDI, well-known esterification and condensation reactions occur and the path followed 
to obtain DiPhAPDI is known as Sono-Gashira reaction method. Synthetic details and structural characterization results are provided at SI and Figures S1-S6.

\subsection{Preparation of organic dye-embedded PS fibers}

PS solution was prepared as $40 \% \mathrm{w} / \mathrm{v}$ in equivolume of Tol. and THF. Then, $1 \mathrm{~mL}$ from this PS solution and $0.3 \mathrm{~mL}$ from the organic dye/THF $(0.2 \mathrm{mg} / \mathrm{mL})$ were mixed in a glass vial. The solution is subjected to electrospinning. The parameters of electrospinning were fixed at $18 \mathrm{kV}$ and $0.7 \mathrm{~mL} \mathrm{~h}^{-1}$ for the flow rate. Due to the potential difference between the tip of syringe and the aluminum foil collector, PS/ organic dye jets were ejected and lead to the formation of PS/organic dye fibers over the aluminum foil. Fibers that were formed in a nonwoven shape over aluminum foil were collected. The starting PS:dye solution is drop-casted and dried under room conditions for comparison.

\subsection{Preparation of PDMS/Organic dye embedded PS fiber composites}

To obtain PDMS/organic dye embedded PS fiber composite freestanding film, collected PS fibers were put in a mold. Then, total amount of $1 \mathrm{~g}$ PDMS, with a ratio of 1:10 for curing agent:oligomer, was cast onto the fiber mats in the mold to cover all fiber surfaces. Mold was put into a vacuum oven at room temperature for $1 \mathrm{~h}$ and then cured at $100^{\circ} \mathrm{C}$ for $15 \mathrm{~min}$. Finally, freestanding PDMS film containing organic dye embedded fiber was extracted from the mold. The entire process is summarized in Scheme 2.

\subsection{WLED studies}

For WLED application, two different preparation methods were used. In the first case, all PS/organic dye fibers were stacked individually with specific amounts and compressed as whole in the mold (Scheme 3a) and labeled as WLED. A single free-standing PDMS/organic dye embedded PS fibers composite film was obtained after casting and curing the PDMS over stacked fibers.

In the second strategy labeled as WLED 2, PDI and DiPhAPDI solutions $(0.2 \mathrm{mg} / \mathrm{mL})$ were mixed in a glass vial that contain $1 \mathrm{~mL}$ from the PS solution ( $40 \% \mathrm{w} / \mathrm{v}$ in equal volume of THF:Tol.) with an equal volume (1:1 v/v ratio). To keep the organic dye/PS ratio that described in Section 2.4, total volume of the PDI and DiPhAPDI mixture was fixed to $0.3 \mathrm{~mL}$. The electrospun fibers that contain equivolume of PDI and DiPhAPDI organic dyes were obtained. These fibers and the already prepared PTE fibers were then stacked in the mold individually with various amounts and free-standing PDMS/organic dye embedded PS fiber composite films were prepared by following the same steps
WLED Sample
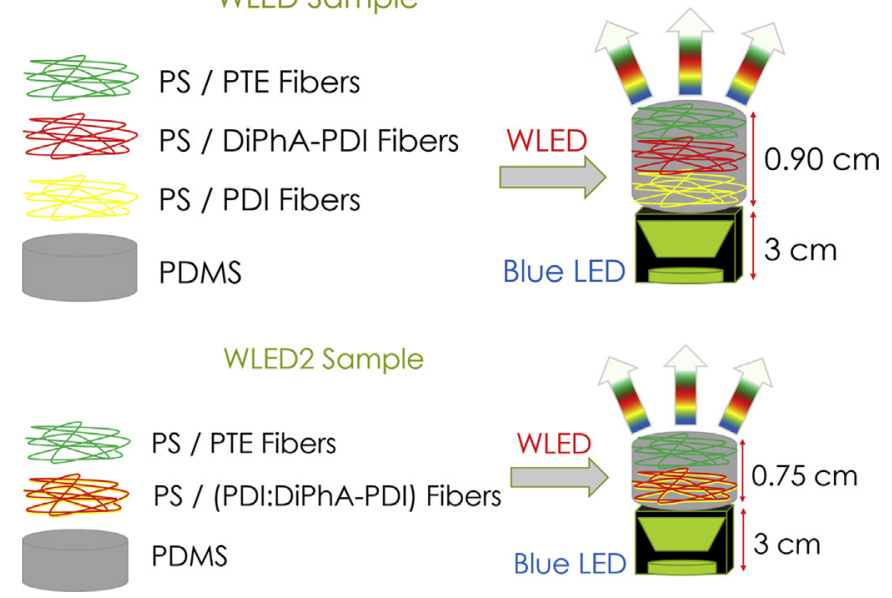

Scheme 3. Visualization of the WLED samples prepared with different processing methods: either by stacking individual fibers or stacking PDI:DiPhAPDI and PTE fibers separately.

described in Section 2.5 (Scheme 3b).

\section{Results \& discussion}

PL properties of the synthesized PDIs in THF:Toluene solution, PS:PDI composites, PS-Fiber, PS-Fiber embedded in PDMS are given in Table 1 and corresponding spectra are provided in Fig. 1. PTE, PDI and DiPhAPDI dyes present PL wavelengths those correspond transitions from S1 to different vibrational levels of S0 and result in blue-green, green-yellow and orange-red colors, respectively.

Emission characteristic of perylene core with tetraester and diimide functionalities are known for many years and the photophysical data obtained for PTE and PDI in solution phase (THF:Tol.) are in good agreement with literature [21,33]. However, extending its emission to longer wavelengths while preventing its extraordinary thermal and photophysical properties is still one of the hot topics of perylene dye research. The most common approach followed in literature is bay functionalization of perylene core of PDI through halogenation reaction followed by attaching amine or phenol derivatives [42]. However, this process causes the perylene ring plane to bend and $\Phi_{\mathrm{PL}}$ to decrease [40]. DiPhAPDI molecule contains phenyl groups attached to the bay positions of PDI through acetyl bridges. This allows the continuity of $\pi$ conjugation while preventing the bending (Figure S7) and as a result PL shifts to longer wavelengths by preserving high $\Phi_{\mathrm{PL}}\left(\Phi_{\mathrm{PL}}{ }^{\mathrm{THF}: \mathrm{Tol}}=95 \%\right)$ (Table 1).

Drop casting method is the most widely used cost effective coating

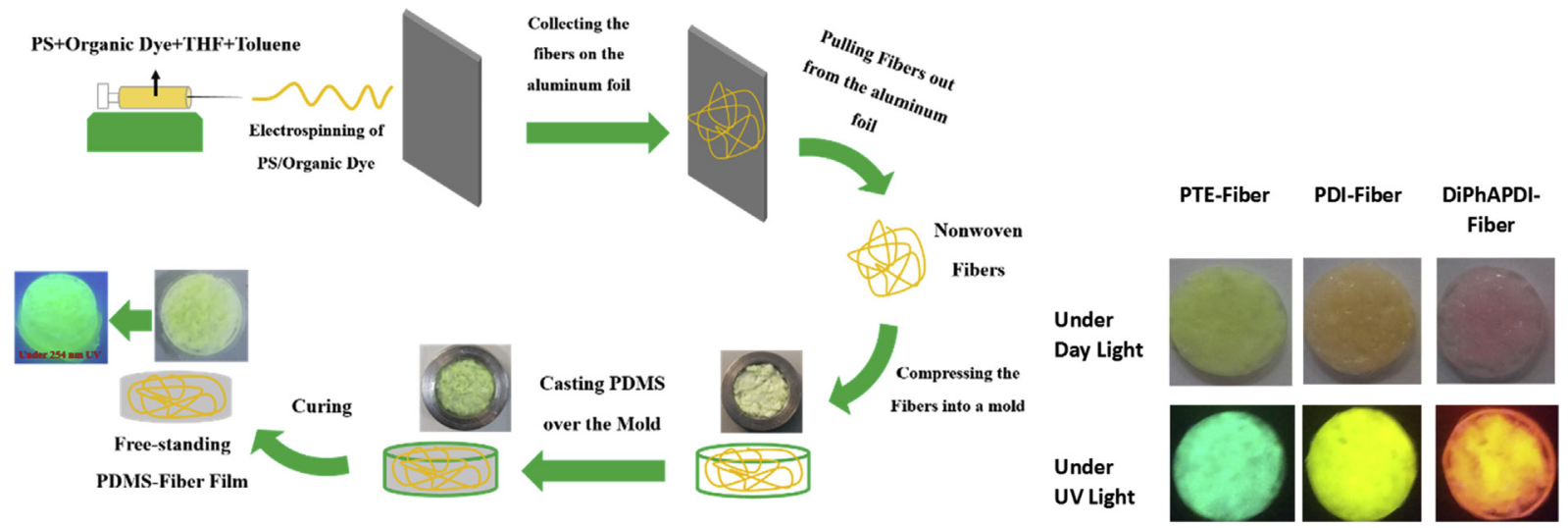

Scheme 2. Summary of electrospinning path followed for PTE, PDI and DiPhAPDI and visualization of their freestanding PDMS films under daylight and UV illumination. 
Table 1

PL and photostability characteristics of PTE, PDI and DiPhAPDI in different media (*photostability data were collected for the 1st PL peaks of the dyes and 455 nm of excitation wavelength, which is the electroluminescence maximum of blue LED, was used.).

\begin{tabular}{|c|c|c|c|c|c|c|c|c|c|c|c|}
\hline \multirow[t]{2}{*}{ Material } & \multirow[t]{2}{*}{ Medium } & \multirow[t]{2}{*}{$\lambda_{\text {exc }}(\mathrm{nm})$} & \multicolumn{3}{|l|}{$\lambda_{\mathrm{PL}}(\mathrm{nm})$} & \multirow[t]{2}{*}{$\varphi_{\mathrm{f}}(\%)$} & \multicolumn{3}{|l|}{$\tau_{\mathrm{AV}}(\mathrm{ns})$} & \multirow[t]{2}{*}{$\mathrm{k}_{\mathrm{p}}^{*}\left(\mathrm{x} 10^{6} \mathrm{~s}^{-1}\right)$} & \multirow[t]{2}{*}{$\mathrm{t}_{1 / 2}{ }^{*}(\mathrm{~h})$} \\
\hline & & & 1st peak & 2nd peak & 3rd peak & & @1st PL peak & @2nd PL peak & @3rd PL peak & & \\
\hline PTE & THF:Tol (1.6:1) & 474 & 488 & 518 & - & 94.0 & 3.9 & 3.9 & - & 5.0 & 38.5 \\
\hline PDI & & 524 & 534 & 574 & 623 & 93.0 & 4.2 & 4.2 & 4.3 & 2.0 & 96.2 \\
\hline DiPhAPDI & & 565 & 593 & 635 & - & 95.0 & 7.3 & 7.1 & 7.2 & 0.6 & 320.8 \\
\hline PTE & Drop-casted & 474 & 505 & 524 & 562 & 58.6 & 5.1 & 8.8 & 7.1 & 20.0 & 9.6 \\
\hline PDI & & 524 & 538 & 576 & 628 & 38.6 & 5.4 & 8.0 & 14.9 & 4.0 & 48.0 \\
\hline DiPhAPDI & & 565 & 591 & 633 & 678 & 33.6 & 7.5 & 13.5 & 20.6 & 0.7 & 275.0 \\
\hline PTE & Fiber & 474 & 496 & 521 & 566 & 84.8 & 4.4 & 5.1 & 6.4 & 0.4 & 481.2 \\
\hline PDI & & 524 & 535 & 575 & 624 & 94.3 & 4.6 & 10.5 & 21.4 & 0.9 & 213.8 \\
\hline DiPhAPDI & & 565 & 589 & 628 & - & 73.6 & 6.6 & 12.5 & - & 0.6 & 320.8 \\
\hline PTE & Fiber in PDMS & 474 & 495 & 520 & - & 73.0 & 4.1 & 4.1 & - & 0.7 & 275.0 \\
\hline PDI & & 524 & 541 & 576 & 625 & 60.0 & 4.94 & 7.96 & 15.25 & 0.3 & 641.6 \\
\hline DiPhAPDI & & 565 & 590 & 635 & - & 52.6 & 6.35 & 8.85 & 12.02 & 0.3 & 641.6 \\
\hline
\end{tabular}

method in investigation of aggregation tendencies and film formation properties of perylene derivatives [33,40]. When the PS:dye solutions are drop casted, although no significant shifts, except for the PTE molecule, are observed in their PL peak positions, the intensity of 2nd and 3rd PL peaks are increased and the $\Phi_{\mathrm{PL}}$ values are decreased. The PL peaks observed for PTE in solution are shifted more than $15 \mathrm{~nm}$ to longer wavelengths. This observation can be attributed to aggregation or possible excimer formation of PTE. The decrement detected in $\Phi_{\mathrm{PL}}$ values of PTE and linear PDI and DiPhAPDI molecules are more than $35 \%$ and $55 \%$, respectively, which addresses $\pi-\pi *$ stacking tendency of all dyes in drop-casted films. Excited state life time profiles of the perylene derivatives in different medium were also measured. The fitting procedure applied to the fluorescence decay profiles yield acceptable statistic $\left(0.8<\mathrm{X}^{2}<1.2\right)$ with a single exponential function for solution and multi exponentials for drop-casted films. Average excited state lifetimes $\left(\tau_{\mathrm{AV}}\right)$ are calculated by using the equation below:

$\tau_{A V}=\frac{\sum_{i=1}^{n} \alpha_{i} \tau_{i}^{2}}{\sum_{i=1}^{n} \alpha_{i} \tau_{i}}$

$\tau_{\mathrm{AV}}$ values of all drop-casted composites are higher than those of their solution phases. The increment is significant for the PS:PDI and PS:DiPhAPDI films. When evaluated with the increase in the intensity of 2nd and 3rd PL peaks, this situation points out aggregation induced emission.

The fibers prepared by electrospinning method of the PS:dye solutions present the best solid state PL properties. Fiber PL curves are similar to the solution phase; however they have higher $\Phi_{\mathrm{PL}}$ values when compared to the drop-casted films. This result indicates that the dyes may be located near the molecular state or mono-state in/on the fibers. It has been reported that even co-polystyrene-derived PDI and their fibers have presented red shifted and broadened PL spectra [15,18] when compared with the PDI core itself. Additionally, the $\Phi_{\mathrm{PL}}$ value reported for this co-polymer was only $53 \%$. Although, no excited state life time measurements were provided, these results, by itself, points out aggregation formation. Herein, we prepared the PS:dye fibers from the monomeric states of the dye molecules and obtained $\Phi_{\mathrm{PL}}$ values are comparable with their solution phases. Although the $\tau_{\mathrm{AV}}$ values of PDI and DiPhAPDI are increased for the 2nd PL peak, one may say that the fibers prepared from the monomeric forms of molecules are more uniform and prevent aggregation to some extent.

When the fibers are embedded in PDMS, although reductions in $\Phi_{\mathrm{PL}}$ values compared to their free-standing fibers are observed, they are still much higher than that of the drop-casted films. The reduction in $\Phi_{\mathrm{PL}}$ values of linear PDI and DiPhAPDI molecules is higher than that of the PTE molecule but the $\Phi_{\mathrm{PL}}$ values are still sufficiently high to enable the downconversion [18].

Photo- and thermal stabilities of organic dyes gain special importance in the life time of the devices they are utilized. Therefore, these parameters of the synthesized molecules in different media are also investigated. Photodegradation rate constants are calculated by using:

$-\ln \left(\frac{I_{0}}{I}\right)=k_{p} t$

where $\mathrm{I}_{0}$ and $\mathrm{I}$ are the emission intensities at times 0 and $t$, respectively and $k_{p}$ is the first order rate constant $\left(s^{-1}\right)$. Half-lives $\left(t_{1 / 2}\right)$ are simply calculated by placing I with I/2. Compared with their solution and drop-casted films the photostabilities of the molecules increased exceptionally (Table 1). Thermograms of PTE, PDI and DiPhAPDI dyes show that the major weight losses start at $360^{\circ} \mathrm{C}, 455^{\circ} \mathrm{C}$ and $455^{\circ} \mathrm{C}$, respectively (Figure S8). The values for PTE and PDI are in good agreement with literature [29,31,43] but DiPhAPDI exhibited approximately $50{ }^{\circ} \mathrm{C}$ higher degradation temperature when compared with other bay functionalized PDI derivatives reported in literature [31]. This situation is attributed to the extended planer conjugation with the acetyl groups. It is known that PS is thermally stable up to $380^{\circ} \mathrm{C}$ [38]; however, the presence of the perylene dyes in PS did not make significant contribution to the thermal stability of PS (Figure S8). Although the degradation curves of PS:dye fibers presented onsets between $370^{\circ} \mathrm{C}$ and $390{ }^{\circ} \mathrm{C}$, these values are still comparable with the widely used inorganic phosphors' and much higher than the detected temperature distribution on LED dies [44-46].

Fig. 2 presents the morphology of the resulting PS:dye fibers. Overall distribution of the composite fibers (Fig. 2a), indicates that fibers are formed individually having the size of $4-6 \mu \mathrm{m}$. The electrospun PS fibers were collected in the form of a belt whose cross-section has Ishape. Moreover, applying higher magnification to these fibers demonstrates that these fibers possess porosity with $50-100 \mathrm{~nm}$ in diameter on their surface (Fig. 2b). The dissolution of PS in a binary solvent system promotes the liq-liq phase separation $[47,48]$. The contrast in vapor pressure of the co-solvents may be the reason of the porous surface feature. THF, which has lower boiling point $\left(66.0^{\circ} \mathrm{C}\right.$ at $\left.1 \mathrm{~atm}\right)$ and higher vapor pressure than that of Tol. $\left(110.6^{\circ} \mathrm{C}\right)$, evaporates faster and leaves pores behind on the fibers' surface. These pores can increase the surface to volume ratio in the fiber compared to that of a one with a smooth surface and therefore, may enhance the interaction of blue light with the fiber. Moreover, these pores can also be considered as individual scattering centers, which again will lead to an enhancement in light-fiber interaction through multiple-scattering of light between fibers. In this sense, due to increasing blue light-fiber interaction porosity 

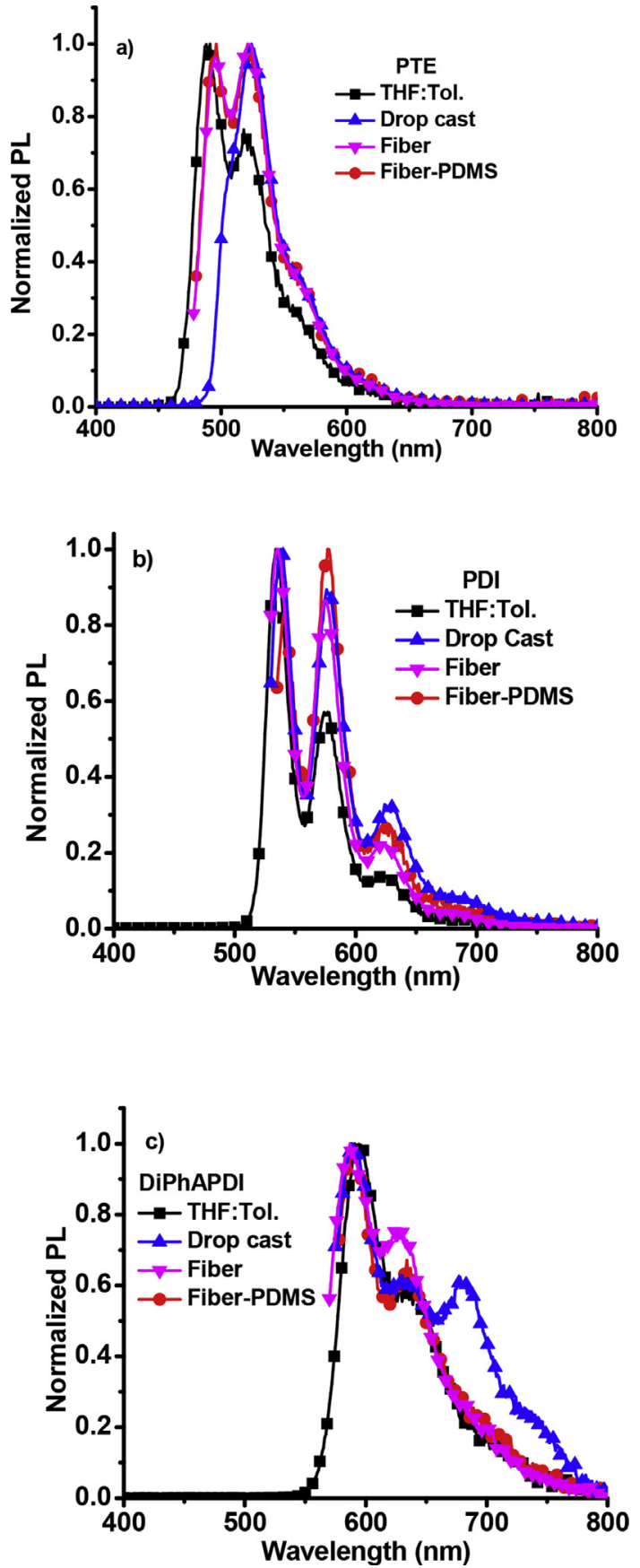

Fig. 1. The PL spectra of PTE, PDI and DiPhAPDI in different media.

may improve the optical path length of the incoming blue light and may enhance the PL of organic dye through multiple excitations. Furthermore, compared to drop-casting of the PS/organic dye solution over a glass surface, which produces a single PS/organic dye film having particular optical features, one can obtain many fibers (approximately $60 \mathrm{mg}$ with the electrospinning setup used in this study) with the same solution by using electrospinning technique. These fibers can be employed in white light applications with different amounts in mass for a single sample to tune the optical features such as CCT and luminous efficiency of the produced frequency conversion layers. Moreover, one can prepare more than one sample by keeping the amount of these fibers fixed. For instance, one can produce three samples from WLED sample 3 or 2 from the WLED 2 sample 2 where they show highly satisfying optical features. Results of these scenarios will be presented and discussed below.
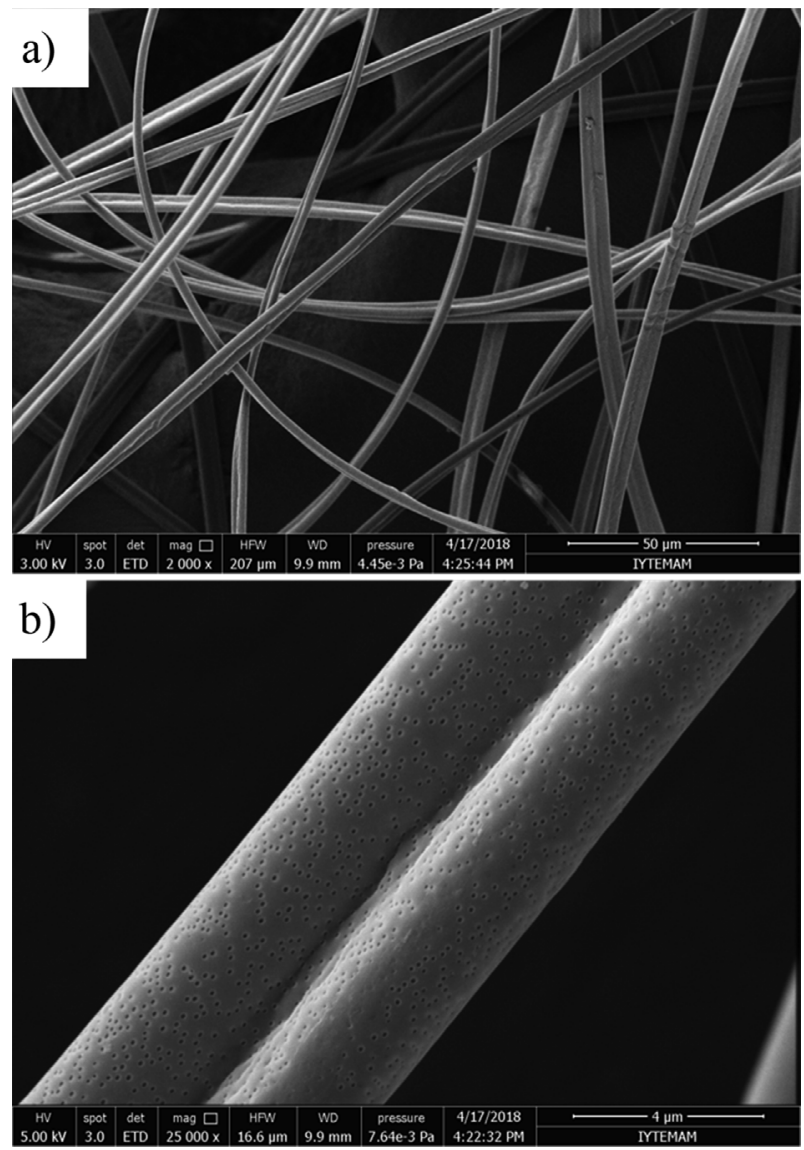

Fig. 2. The morphology of PS/organic dye composite fibers.

Prepared organic dye embedded PS fibers with different colors, PTE as green, PDI as yellow, and DiPhAPDI as red, were put into a form of color conversion layer by casting PDMS over these fibers in the mold, as already described in Scheme 1. PDMS/PTE, PDI, and DiPhAPDI composite fibers were placed onto a $455 \mathrm{~nm}$ blue LED under driving current of $20 \mathrm{~mA}$ separately first. Fig. 3a presents the resulting spectrum of these samples. Obviously, PDI and PTE show explicit emission under blue LED and maintain their individual characteristic PL signals. On the other hand, red emission of the DiPhAPDI sample is not efficient in terms of PL intensity as others, but still can contribute as red color to overall system. Fig. 3b presents PL spectrum of the WLED samples described in Section 2.6 with varying individual fiber masses. Note that stacking sequence of these individual organic dye fibers, corresponding to PTE, PDI and DiPhAPDI dyes separately, was determined by considering their absorption and quantum yield features. In the case of preparing conversion layers with stacking formation, for instance, stacking red and yellow phosphors one after the other, the mainstream design is to sort these individual conversion layers from lowest bandgap to highest above LED to avoid from internal absorptions. Therefore, DiPhAPDI, PDI and PTE fibers are expected to be stacked over blue LED in this order. However, even though PTE fibers are still the farthest from the LED, locations of DiPhAPDI and PDI are replaced to increase PL intensity of DiPhAPDI fibers based on internal absorption such as its additional absorption from PDI. It is observed from their spectrum that the individual characteristic peaks of the organic dyes are still preserved even after their stacking formation and PDMS coating for all samples. In terms of optical features, all samples were achieved to show CRI greater than 85 (Table 2). From Sample 1 to Sample 3, as the total fiber mass increases, CCT started to reduce from $6679 \mathrm{~K}$ to $4918 \mathrm{~K}$ while CRI shows a clear increment from 86 to 92 , which is based on the improved red-emission due to increasing amount of DiPhAPDI fiber. 

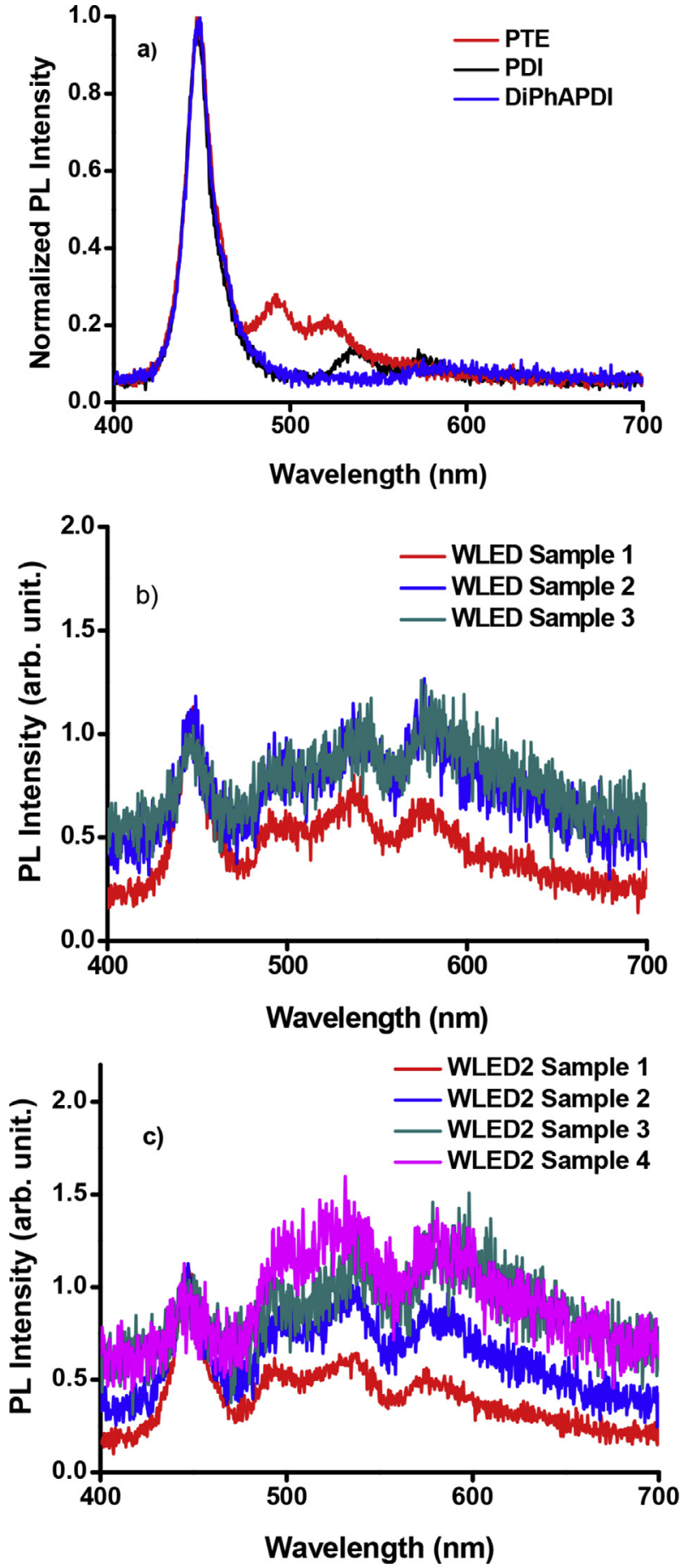

Fig. 3. PL spectrum of individual PDMS/organic dye electrospun fiber composites and their various formation strategies including WLED and WLED 2 (driving current $=20 \mathrm{~mA}$ ).

Table 2

Fiber masses and optical properties of the related samples used in WLED strategy under $20 \mathrm{~mA}$ of driving current.

\begin{tabular}{|c|c|c|c|c|c|c|}
\hline Sample\# & $\begin{array}{l}\text { PDI } \\
\text { Fiber } \\
\text { Mass } \\
\text { (mg) }\end{array}$ & $\begin{array}{l}\text { DiPhAPDI } \\
\text { Fiber Mass } \\
(\mathrm{mg})\end{array}$ & $\begin{array}{l}\text { PTE } \\
\text { Fiber } \\
\text { Mass } \\
(\mathrm{mg})\end{array}$ & CRI & ССТ (K) & $\begin{array}{l}\text { Power } \\
\text { efficiency } \\
(\mathrm{lm} / \mathrm{W})\end{array}$ \\
\hline 1 & 15 & 10 & 10 & 86 & 6679 & 222 \\
\hline 2 & 20 & 15 & 10 & 89 & 5205 & 212 \\
\hline 3 & 20 & 20 & 10 & 92 & 4918 & 197 \\
\hline
\end{tabular}

Table 3

Fiber masses and optical properties of the related samples used in WLED2 strategy under $20 \mathrm{~mA}$ of driving current.

\begin{tabular}{llllll}
\hline Sample\# & $\begin{array}{l}\text { PDI:DiPhAPDI Fiber } \\
\text { Mass (mg) }\end{array}$ & $\begin{array}{l}\text { PTE Fiber } \\
\text { Mass (mg) }\end{array}$ & CRI & CCT (K) & $\begin{array}{l}\text { Power } \\
\text { efficiency (lm/ } \\
\text { W) }\end{array}$ \\
\hline 1 & 22 & 6 & 89 & 7490 & 219 \\
2 & 30 & 10 & 89 & 5677 & 220 \\
3 & 40 & 10 & 94 & 4646 & 199 \\
4 & 35 & 15 & 90 & 5102 & 211 \\
\hline
\end{tabular}

Therefore, with the adjustment of fiber content, one can achieve improvement in CRI and tune the CCT values. However, there is an opposite correlation between total fiber content, CRI and luminous efficiency. With the increasing CRI together with increasing fiber content, luminous efficiencies show a slight decrease. For the second strategy (WLED 2) that is described in Section 2.6, resulting PL spectrum of WLED 2 samples is given in Fig. 3c. Even though the contribution of red and yellow colors are now coming from a single fiber composition unlike the first strategy where these dyes contribute individually based on their sequential positioning as two different fiber contents, it is observed that the resulting PL still contains the individual characteristic PL peaks of these dyes. Similarly, by varying the amount of fibers in mass, different CRI values are obtained. All samples showed values of CRI $\geq 90$, and adjustable CCT values that are taking values between $7500 \mathrm{~K}$ and $5000 \mathrm{~K}$ (Table 3). Meanwhile, luminous efficiency again drops approximately $201 \mathrm{~m} / \mathrm{W}$. One further sample, as WLED 2 sample 4, was produced with using the same total fiber amount in mass with WLED 2 sample 3 to compare the effect of PDI: DiPhAPDI and PTE fiber content ratios. It is clear that even though they have the same total fiber mass in total, by increasing the PTE amount while decreasing the PDI: DiPhAPDI lead to a decrease in CRI and an increase in both CCT and luminous efficiency. Therefore, one can conclude that even though total fiber amount is significant in the fabrication of WLED products containing these PS/organic dye fibers to obtain desired optical features, the ratio of employed fibers in either WLED or WLED 2 strategies can also provide a fine tuning among these optical features.

Fig. 4 presents the change of optical properties such as power efficiency, CRI, and CCT with respect to driving current. In the first case, there is a clear inverse relationship between increasing the driving current and the power efficiencies of individual PDMS/organic dye fiber composites including PTE, PDI, and DiPhAPDI dyes in the order based on their quantum yields from highest to lowest. Comparing these samples with bare LED output, which shows a dramatic decrease with the increasing driving current, reveals that even though these samples have decreasing profile for power efficiencies, are still less than the bare LED output. Among those samples, PDMS/PTE fiber composite remains almost stable. On the other hand, WLED and WLED 2 samples, which were selected among the ones with highest CRI; Sample 3 of both strategies indicate dramatic increase with the increasing driving current. They follow almost the same path together, and reach even $3001 \mathrm{~m} / \mathrm{W}$ of power efficiency at higher driving current values. Further investigation was performed for these WLED and WLED 2 samples in terms of their CRI and CCT values. Obviously, CRI and CCT values of the both samples decrease as the driving current is increased. In the case of CRI, among the decaying profiles of these samples, WLED 2 has the lowest decaying rate. However, in the case of CCT, WLED 2 decays faster than the WLED unlike the previous case, providing a broader tunability range for the CCT values. Therefore, WLED 2 can be offered as a better strategy than the WLED since its CRI values drop less with the increasing driving current while CCT values show a broader range of adjustment.

Another essential parameter that determines whether these samples can be commercialized or not is their optical stability against continuous illumination of blue LED. In this sense, periodic measurements 

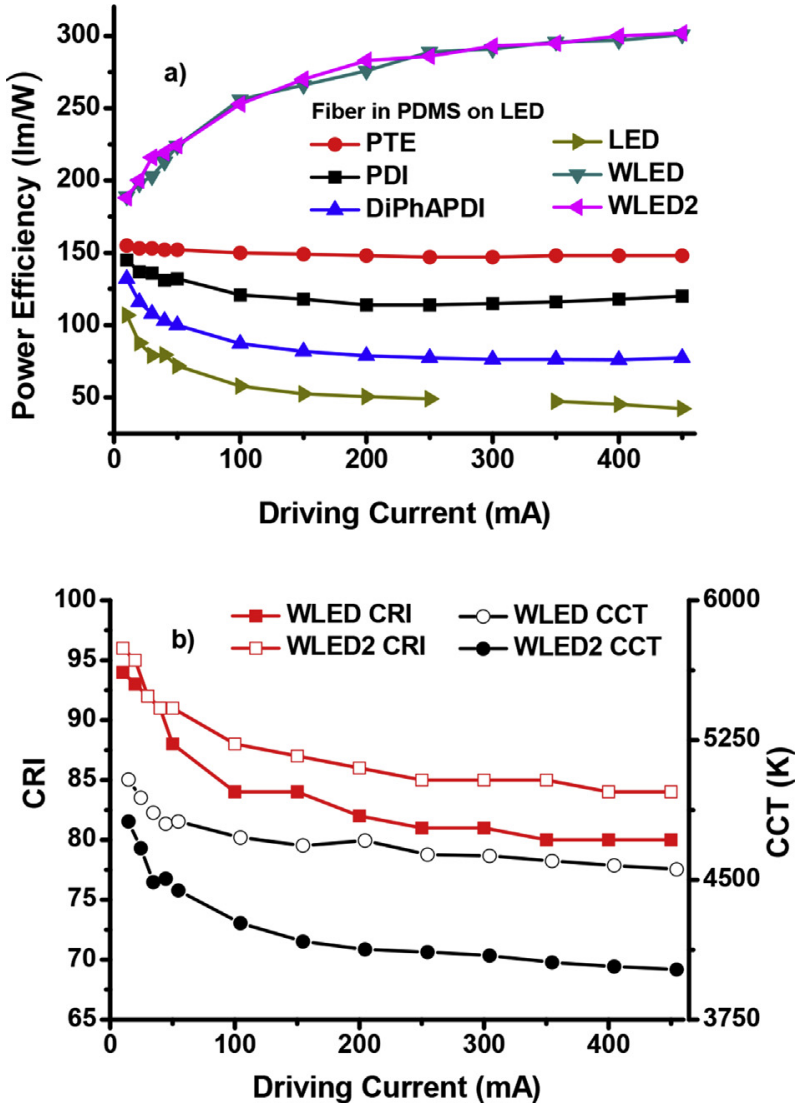

Fig. 4. Change of optical properties of a) power efficiency and b) CRI and CCT under various driving currents.

versus time have been performed on the same WLED and WLED 2 samples that were used already in previous case. The results are given in Fig. 5. It is observed that even though there is a fluctuation for the values of power efficiencies of both WLED and WLED 2 samples, both datasets can be fitted to a linearly straight curve showing a clear stability in time (for $240 \mathrm{~min}$ ). These samples have almost the same fitting curves indicating that both samples show the same stability performance in terms of their power efficiencies against continuous illumination. On the other hand, CRI and CCT values of both samples were observed to follow a linear straight curve also in time similar to previous case. The main difference between these two samples is their different CRI and CCT values that may be the reason of the shift observed for both $y$-axis of panel $b$ unlike the power efficiencies where they were started from almost the same point in their y-axis. However, even though these straight stability curves have different starting points, they indicate an obvious stability against continuous illumination. In this sense, it can be concluded that introduced PS:dye fibers embedded in PDMS matrices can be promising alternatives to inorganic phosphors as frequency conversion layer to be used in lighting industry in future.

\section{Conclusion}

The photophysical properties of green (PTE), yellow (PDI) and red (DiPhAPDI) emitting perylene derivatives in solution, drop-casted films, PS fibers and PS fibers embedded in PDMS are presented. In DiPhAPDI molecule PL wavelength is shifted to longer wavelengths compared to regular PDI derivatives and the $\Phi_{\mathrm{f}}$ is prevented. In their PS fibers, the used perylene derivatives not only presented exceptional photostabilities but also preserved their extraordinary photophysical properties. By using the advantage of this result, a fabrication method of organic dye based frequency conversion layers to be used in WLED
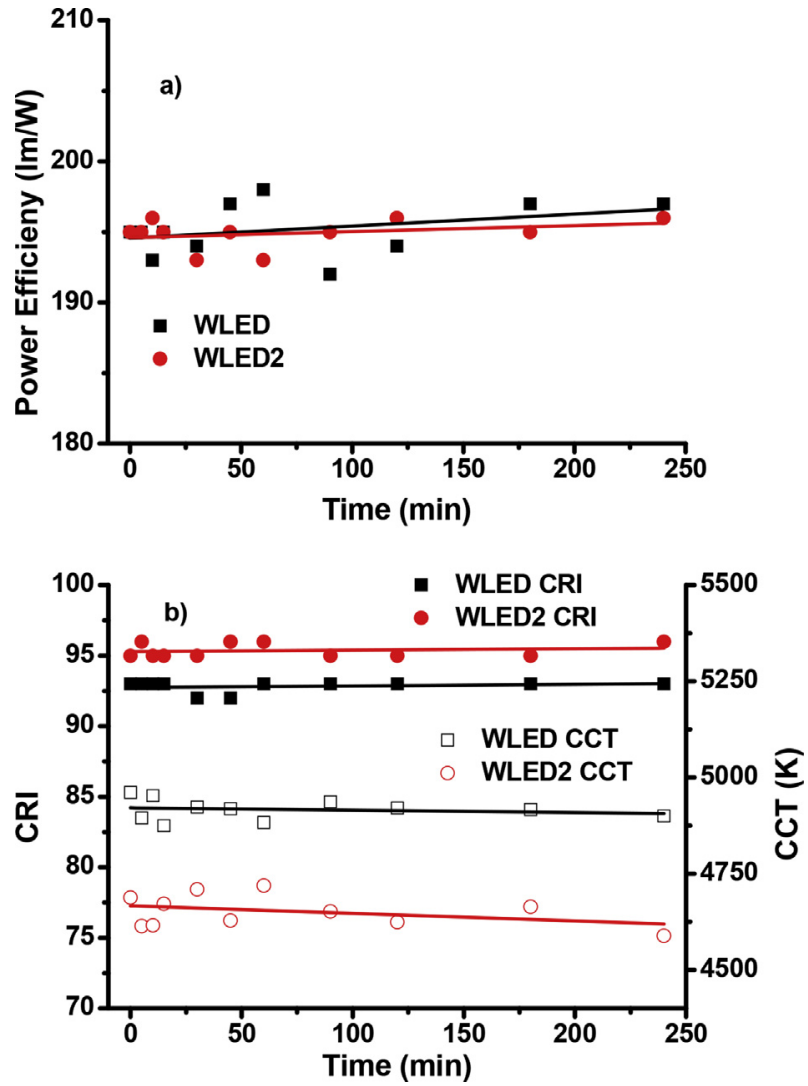

Fig. 5. Change of power efficiency, CRI and CCT under continuous illumination of blue LED (driving current $=20 \mathrm{~mA}$ ). (For interpretation of the references to color in this figure legend, the reader is referred to the Web version of this article.)

Table 4

Performance summary of literature that the perylene dyes were used as down conversion materials.

\begin{tabular}{|c|c|c|c|c|c|}
\hline $\begin{array}{l}\text { Coating } \\
\text { Method }\end{array}$ & Host & CIE (x;y) & CRI & $\begin{array}{l}\text { Power efficiency } \\
\text { (lm/W@20 mA) }\end{array}$ & Ref. \\
\hline Drop casting & PMMA & $0.34 ; 0.29$ & 90 & 8.5 & [40] \\
\hline Spin coating & PMMA & $0.30 ; 0.39$ & - & - & [19] \\
\hline Dip-coated & PMMA & $0.35 ; 0.34$ & 84 & 126 & [16] \\
\hline Spin coated & PMMA & - & - & 67 & [17] \\
\hline Dip coating & & $0.26 ; 0.36$ & - & 118 & \\
\hline Spin coating & PS-copolymer & $0.31 ; 0.34$ & 83 & 28 & [15] \\
\hline Nanofiber & PS-copolymer & $0.36 ; 035$ & 65 & 70 & [18] \\
\hline Nanofiber & $\begin{array}{l}\text { PS-Fiber } \\
\text { embedded in } \\
\text { PDMS }\end{array}$ & $0.36 ; 0.36$ & 94 & 199 & $\begin{array}{l}\text { This } \\
\text { work }\end{array}$ \\
\hline
\end{tabular}

applications is offered. Commission Internationale de l'Eclairage (CIE) graphs (Fig. S9) showed that all WLED samples are located at the center. To the best of our knowledge, fabricated samples showed the highest CRI and power efficiency values (Table 4) in literature that the perylene dyes were used as down conversion materials and adjustable CCT depending on the used fiber amount in mass. Moreover, applying various driving currents and exposing these samples to continuous illumination of blue LED reveal that these samples have the potential of utilization as frequency down conversion layers.

\section{Acknowledgements}

The authors thank to IZTECH-Center for Materials Research for microscopy imaging. 


\section{Appendix A. Supplementary data}

Supplementary data related to this article can be found at https:// doi.org/10.1016/j.dyepig.2018.08.040.

\section{References}

[1] Mosca C, Mauro, Caruso Fulvio, Zambito Leandro, Macaluso Roberto, Cal1. Hybrid LEDs pave way to new lighting applications. Photonics Spectra; 2013.

[2] U.S. Department of Energy (Energy Efficiency and Renewable Energy). Solid state lighting research and development. Washington D.C. USA: Multi Year Program Plan; 2011.

[3] Cho J, Park JH, Kim JK, Schubert EF. White light-emitting diodes: history, progress, and future. Laser Photon Rev 2017;11:1600147. https://doi.org/10.1002/lpor. 201600147.

[4] Shinde KN, Dhoble SJ. Europium-activated orthophosphate phosphors for energyefficient solid-state lighting: a review. Crit Rev Solid State Mater Sci 2014;39:459-79. https://doi.org/10.1080/10408436.2013.803456.

[5] Goretzki G, Davies ES, Argent SP, Warren JE, Blake AJ, Champness NR. Building multistate redox-active architectures using metal-complex functionalized perylene bis-imides. 2009. p. 10264-74. https://doi.org/10.1021/ic901379d.

[6] Narendran N, Gu Y, Freyssinier-Nova JP, Zhu Y. Extracting phosphor-scattered photons to improve white LED efficiency. Phys. Status Solidi Appl. Mater. Sci. 2005;202:60-2. https://doi.org/10.1002/pssa.200510015.

[7] Güner T, Şentürk U, Demir MM. Optical enhancement of phosphor-converted wLEDs using glass beads. Opt Mater 2017;72:769-74. https://doi.org/10.1016/j. optmat.2017.07.033.

[8] Güner T, Köseoğlu D, Demir MM. Multilayer design of hybrid phosphor film for application in LEDs. Opt Mater 2016;60:422-30. https://doi.org/10.1016/j.optmat. 2016.08.023.

[9] Xia Z, Xu Z, Chen M, Liu Q. Recent developments in the new inorganic solid-state LED phosphors. Dalton Trans 2016;45:11214-32. https://doi.org/10.1039/ C6DT01230B.

[10] George NC, Denault KA, Seshadri R. Phosphors for solid-state white lighting. Annu Rev Mater Res 2013;43:481-501. https://doi.org/10.1146/annurev-matsci073012-125702.

[11] Zhu H, Lin CC, Luo W, Shu S, Liu Z, Liu Y, et al. Highly efficient non-rare-earth red emitting phosphor for warm white light-emitting diodes. Nat Commun 2014;5. https://doi.org/10.1038/ncomms5312.

[12] Chen D, Zhou Y, Zhong J. A review on $\mathrm{Mn}^{4+}$ activators in solids for warm white light-emitting diodes. RSC Adv 2016;6:86285-96. https://doi.org/10.1039/ C6RA19584A.

[13] Guner T, Demir MM. A review on halide perovskites as color conversion layers in white light emitting diode applications. Phys Status Solidi 2018;1800120:1800120. https://doi.org/10.1002/pssa.201800120.

[14] He X, Qiu Y, Yang S. Fully-inorganic trihalide perovskite nanocrystals: a new research frontier of optoelectronic materials. Adv Mater 2017;29:1-27. https://doi. org/10.1002/adma.201700775.

[15] Kozma E, Mróz W, Galeotti F. A polystyrene bearing perylene diimide pendants with enhanced solid state emission for white hybrid light-emitting diodes. Dyes Pigments 2015;114:138-43. https://doi.org/10.1016/j.dyepig.2014.11.009.

[16] Mosca M, Caruso F, Zambito L, Seminara B, Macaluso R, Calì C, et al. Warm white LED light by frequency down-conversion of mixed yellow and red Lumogen. Proc SPIE-Int Soc Opt Eng 2013;8767:87670L. https://doi.org/10.1117/12.2017274.

[17] Caruso F, Mosca M, Macaluso R, Feltin E, Calì C. Generation of white LED light by frequency downconversion using perylene-based dye. Electron Lett 2012;48:1417-9. https://doi.org/10.1049/el.2012.3084.

[18] Galeotti F, Mróz W, Catellani M, Kutrzeba-Kotowska B, Kozma E. Tailorable perylene-loaded fluorescent nanostructures: a multifaceted approach enabling their application in white hybrid LEDs. J Mater Chem C. 2016;4:5407-15. https://doi. org/10.1039/C6TC00486E.

[19] Caruso F, Mosca M, Rinella S, Macaluso R, Calì C, Saiano F, et al. Frequencydownconversion stability of PMMA coatings in hybrid white light-emitting diodes. J Electron Mater 2015;45:682-7. https://doi.org/10.1007/s11664-015-4173-y.

[20] Maiti DK, Bhattacharjee R, Datta A, Banerjee A. Modulation of fluorescence resonance energy transfer efficiency for white light emission from a series of stilbeneperylene based donor-acceptor pair. J Phys Chem C 2013;117:23178-89. https:// doi.org/10.1021/jp409042p.

[21] Zafer C, Karapire C, Serdar NS, Icli S, Serdar Sariciftci N, Icli S. Characterization of N, N'-bis-2-(1-hydoxy-4-methylpentyl)-3, 4, 9, 10-perylene bis (dicarboximide) sensitized nanocrystalline TiO2 solar cells with polythiophene hole conductors. Sol Energy Mater Sol Cells 2005;88:11-21. https://doi.org/10.1016/j.solmat.2004.09. 009.

[22] Fan W, Liang N, Meng D, Feng J, Li Y, Hou J, et al. A high performance threedimensional thiophene-annulated perylene dye as an acceptor for organic solar cells. Chem Commun 2016;52:11500-3. https://doi.org/10.1039/C6CC05810H.

[23] Chen W, Yang X, Long G, Wan X, Chen Y, Zhang Q. A perylene diimide (PDI)-based small molecule with tetrahedral configuration as a non-fullerene acceptor for organic solar cells. J Mater Chem C. 2015;3:4698-705. https://doi.org/10.1039/ C5TC00865D.

[24] Li S, Liu W, Li C-Z, Lau T-K, Lu X, Shi M, et al. A non-fullerene acceptor with a fully fused backbone for efficient polymer solar cells with a high open-circuit voltage. J Mater Chem A. 2016;4:14983-7. https://doi.org/10.1039/C6TA07368A.

[25] Liang N, Sun K, Zheng Z, Yao H, Gao G, Meng X, et al. Perylene diimide trimers based bulk heterojunction organic solar cells with efficiency over $7 \%$. Adv. Energy Mater. 2016;6. https://doi.org/10.1002/aenm.201600060.

[26] Jiang W, Ye L, Li X, Xiao C, Tan F, Zhao W, et al. Bay-linked perylene bisimides as promising non-fullerene acceptors for organic solar cells. Chem Commun 2014;50:1024-6. https://doi.org/10.1039/c3cc47204c.

[27] Liu X, Cai Y, Huang X, bo Zhang R, Sun X. A perylene diimide electron acceptor with a triptycene core for organic solar cells. J Mater Chem C. 2017;5:3188-94. https://doi.org/10.1039/C7TC00378A.

[28] Li C, Wonneberger H. Perylene imides for organic photovoltaics: yesterday, today, and tomorrow. Adv Mater 2012;24:613-36. https://doi.org/10.1002/adma. 201104447.

[29] Huang C. Perylene diimide-based materials for organic electronics and optical limiting applications. Georgia Institute of Technology; 2010.

[30] Oner I, Varlikli C, Icli S. The use of a perylenediimide derivative as a dopant in hole transport layer of an organic light emitting device. Appl Surf Sci 2011;257:6089-94. https://doi.org/10.1016/j.apsusc.2011.02.002.

[31] Kozma E, Kotowski D, Luzzati S, Catellani M, Bertini F, Famulari A, et al. Improving the efficiency of P3HT:perylene diimide solar cells via bay-substitution with fused aromatic rings. RSC Adv 2013;3:9185-8. https://doi.org/10.1039/c3ra41574k.

[32] Zhao H, Zhang YY, Xu H, He ZM, Zhang ZL, Zhang HQ. Synthesis and properties of perylene diimide dyes bearing unsymmetrical and symmetrical phenoxy substituents at bay positions. Tetrahedron 2015;71:7752-7. https://doi.org/10.1016/j. tet.2015.07.032.

[33] Karapire C, Timur C, İçli S. A comparative study of the photophysical properties of perylenediimides in liquid phase, PVC and sol-gel host matrices. Dyes Pigments 2003;56:135-43. https://doi.org/10.1016/S0143-7208(02)00128-6.

[34] Mameri S, Siekierzycka JR, Brouwer AM. Artificial miniaturized luminescent materials based on perylene-covered glass surfaces. New J Chem 2017;41:6083-8. https://doi.org/10.1039/c7nj01337j.

[35] Demir MM, Horzum N, Özen B, Özçelik S. Hierarchial coassembly of a cyanine dye in poly(vinyl alcohol) fibrous films by electrospinning. J Phys Chem B 2013;117:10920-8. https://doi.org/10.1021/jp404977d.

[36] Electrospinning F, Link C. formation of pseudoisocyanine J-aggregates in poly ( vinyl alcohol ). 2013. p. 11568-73.

[37] Demir MM, Yilgor I, Yilgor E, Erman B. Electrospinning of polyurethane ${ }^{\circledR}$ bers 2002;43:3303-9.

[38] Sherif SA, Abdel-Halim MS, El-Marsafy SM, El-Sayed MH. Thermal degradation of polystyrene. J Eng Appl Sci 2003;50:587-602. https://doi.org/10.1016/S01652370(00)00159-5.

[39] Radhakrishnan TS. Thermal degradation of poly(dimethylsilylene) and poly(tetramethyldisilylene-co-styrene). J Appl Polym Sci 2006;99:2679-86. https://doi.org/ 10.1002/app.22813.

[40] E. Aksoy, N. Demir, C. Varlikli, White LED light production by using dibromoperylene derivatives in down conversion of energy, Can J Phys 1-16. doi:https://doi. org/10.1139/cjp-2017-0752.

[41] Wang Y, Zhang L, Zhang G, Wu Y, Wu S, Yu J, et al. A new colorimetric and fluorescent bifunctional probe for $\mathrm{Cu} 2+$ and $\mathrm{F}$ - ions based on perylene bisimide derivatives. Tetrahedron Lett 2014;55:3218-22. https://doi.org/10.1016/j.tetlet. 2014.03.137.

[42] Dubey RK, Efimov A, Lemmetyinen H. 1,7-And 1,6-regioisomers of diphenoxy and dipyrrolidinyl substituted perylene diimides: synthesis, separation, characterization, and comparison of electrochemical and optical properties. Chem Mater 2011;23:778-88. https://doi.org/10.1021/cm1018647.

[43] Karapire C, Zafer C, İçli S. Studies on photophysical and electrochemical properties of synthesized hydroxy perylenediimides in nanostructured titania thin films. Synth Met 2004;145:51-60. https://doi.org/10.1016/j.synthmet.2004.04.016.

[44] Yang TH, Huang HY, Sun CC, Glorieux B, Lee XH, Yu YW, et al. Noncontact and instant detection of phosphor temperature in phosphor-converted white LEDs. Sci Rep 2018;8:1-10. https://doi.org/10.1038/s41598-017-18686-z.

[45] Tian Y. Development of phosphors with high thermal stability and efficiency for phosphor-converted LEDs. J. Solid State Light. 2014;1:11. https://doi.org/10.1186/ s40539-014-0011-8.

[46] W. Liu, D. Wu, H. Chang, R. Duan, W. Wu, G. Amu, et al., The enhanced red emission and improved thermal stability of CaAlSiN 3 : Eu $2+$ phosphors by using nano-EuB 6 as raw material, (n.d.). doi:http://doi.org/10.3390/nano8020066.

[47] Demir MM, Wegner G. Challenges in the preparation of optical polymer composites with nanosized pigment particles: a review on recent efforts. Macromol Mater Eng 2012;297:838-63. https://doi.org/10.1002/mame.201200089.

[48] Demir MM. Investigation on glassy skin formation of porous polystyrene fibers electrospun from DMF. Express Polym Lett 2010;4:2-8. https://doi.org/10.3144/ expresspolymlett.2010.2. 